\title{
Fluorescence Correlation Spectroscopy, a Tool to investigate Supramolecular Dynamics: Inclusion Complexes of Pyronines with Cyclodextrin.
}

\author{
Wajih Al-Soufi*a, Belén Reija ${ }^{a}$, Mercedes Novo ${ }^{a}$, \\ Suren Felekyan $^{b}$, Ralf Kühnemuth ${ }^{b}$ and Claus A. M. Seidel ${ }^{b}$ \\ a Departamento de Química Física, Facultade de Ciencias, \\ Universidade de Santiago de Compostela, E-27002 Lugo (Spain) \\ ${ }^{b}$ Lehrstuhl für Molekulare Physikalische Chemie, \\ Heinrich-Heine Universität Düsseldorf, Universitätsstraße 1, \\ D-40225 Düsseldorf (Germany) \\ E-mail: alsoufi@lugo.usc.es

\section{Supplementary Information}

\section{Number of collisions during the lifetime of an encounter complex}

The number of collisions $N_{\text {coll }}$ may be estimated roughly assuming that the molecules within the solvent cage have a mean speed $\bar{v}$ similar to that in the gas phase.

From Maxwell's kinetic gas theory we calculate a mean speed of the molecules

$$
\overline{\mathrm{v}}=\left(\frac{8 k T}{\pi \mu}\right)^{\frac{1}{2}}
$$

at temperature $T$ and a reduced mass $\mu$ of the encounter complex.

Assuming a mean free path $\bar{\lambda}$ in the encounter complex of the order of the distance between the molecular centres $\bar{\lambda} \approx R_{A H}$ we obtain a collision frequency $v$

$$
v=\frac{\overline{\mathrm{v}}}{\bar{\lambda}} \approx \frac{\overline{\mathrm{v}}}{R_{A H}}
$$

With the lifetime of the encounter complex

$$
t_{\text {enc }}=\left(k_{-d}\right)^{-1}=\frac{R_{A H}^{2}}{4 D_{A H}}
$$

we estimate finally the number of collisions

$$
N_{\text {coll }}=v \cdot t_{\text {enc }} \approx \frac{\overline{\mathrm{v}}}{R_{A H}} t_{\text {enc }}=\frac{R_{A H}}{4 D_{A H}}\left(\frac{8 k T}{\pi \mu}\right)^{\frac{1}{2}}
$$

(See Caldin, E.F. The mechanism of fast reactions in solution. IOS Press, Amsterdam, 2001) 


\section{Estimation of the fraction of successful collisions between guest and host.}

The probability $P_{r}$ that host and guest form an inclusion complex at each of the collisions in the encounter complex can be estimated as ratio $f_{r}$ between the total number $N$ of possible relative orientations between host and guest and the number $N_{r}$ of those orientations which lead to a successful inclusion of the guest in the host:

$$
f_{r}=\frac{N_{r}}{N}
$$

We suppose that host and guest collide randomly. All orientations have equal probability. Successive collisions are taken as independent and no specific interactions are taken into account. The inclusion depends only on geometric conditions.

\section{Geometric description}

The molecules are approximated as rectangular prisms with faces $b \times c$ and length $L$ :
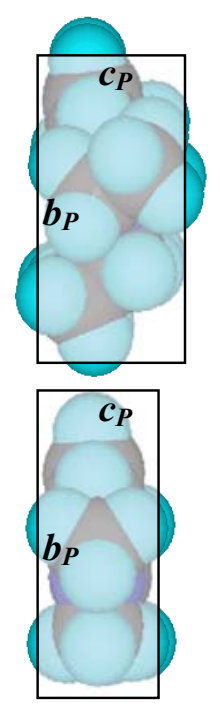
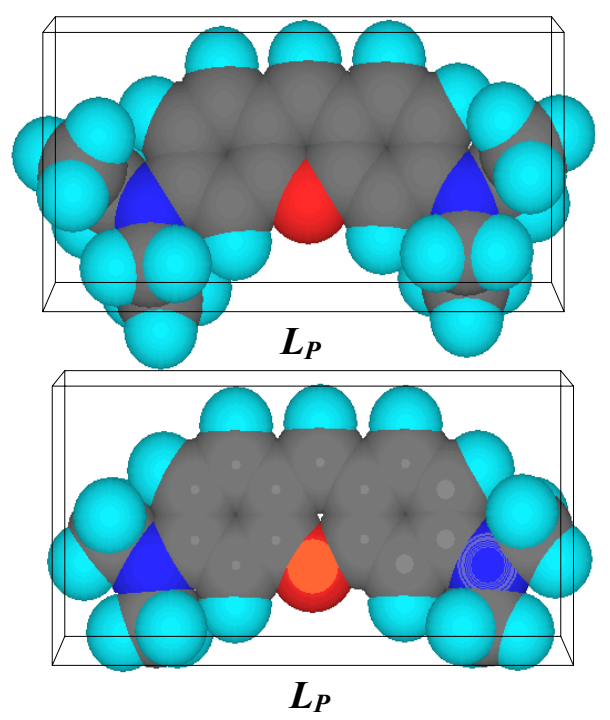

PB

PY

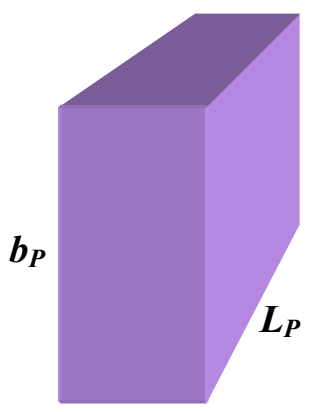

$\boldsymbol{c}_{P}$
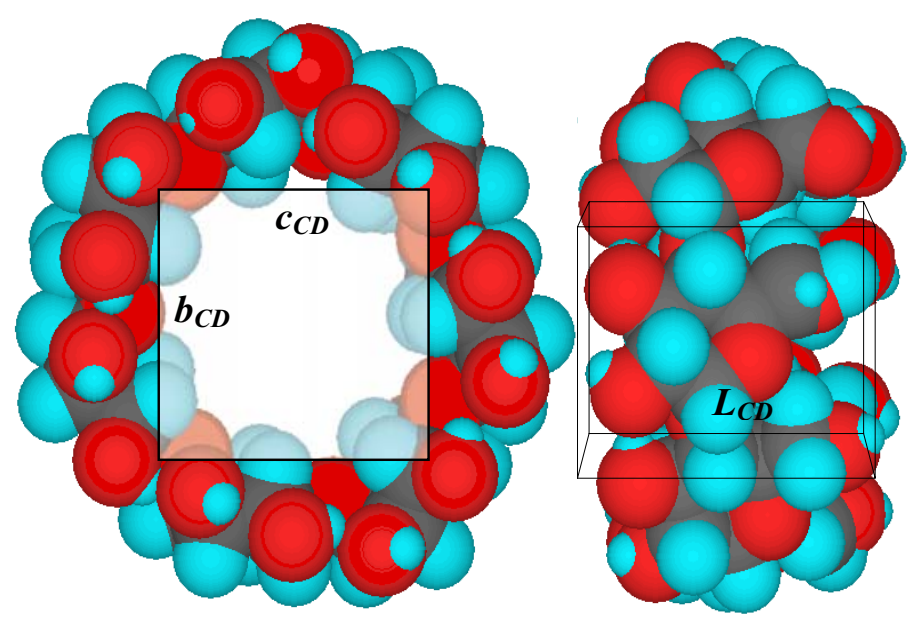

$\beta-C D \quad b_{C D}$

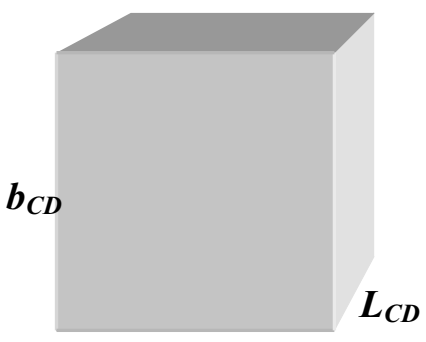

$c_{C D}$

Figure S1: Geometric description of guest and host. 
In the instance of a collision the relative position of host and guest is described by four angles $\alpha, \beta, \delta$ and $\gamma$ with respect to a ,collision axis" through the centers of the molecules. The angles $\alpha, \beta$ are inclination angles, $\delta$ gives the relative rotation between host and guest and $\gamma$ the rotation of the guest around its longer molecular axis. $\alpha=\beta=\delta=0$ corresponds to a perfectly linear orientation.
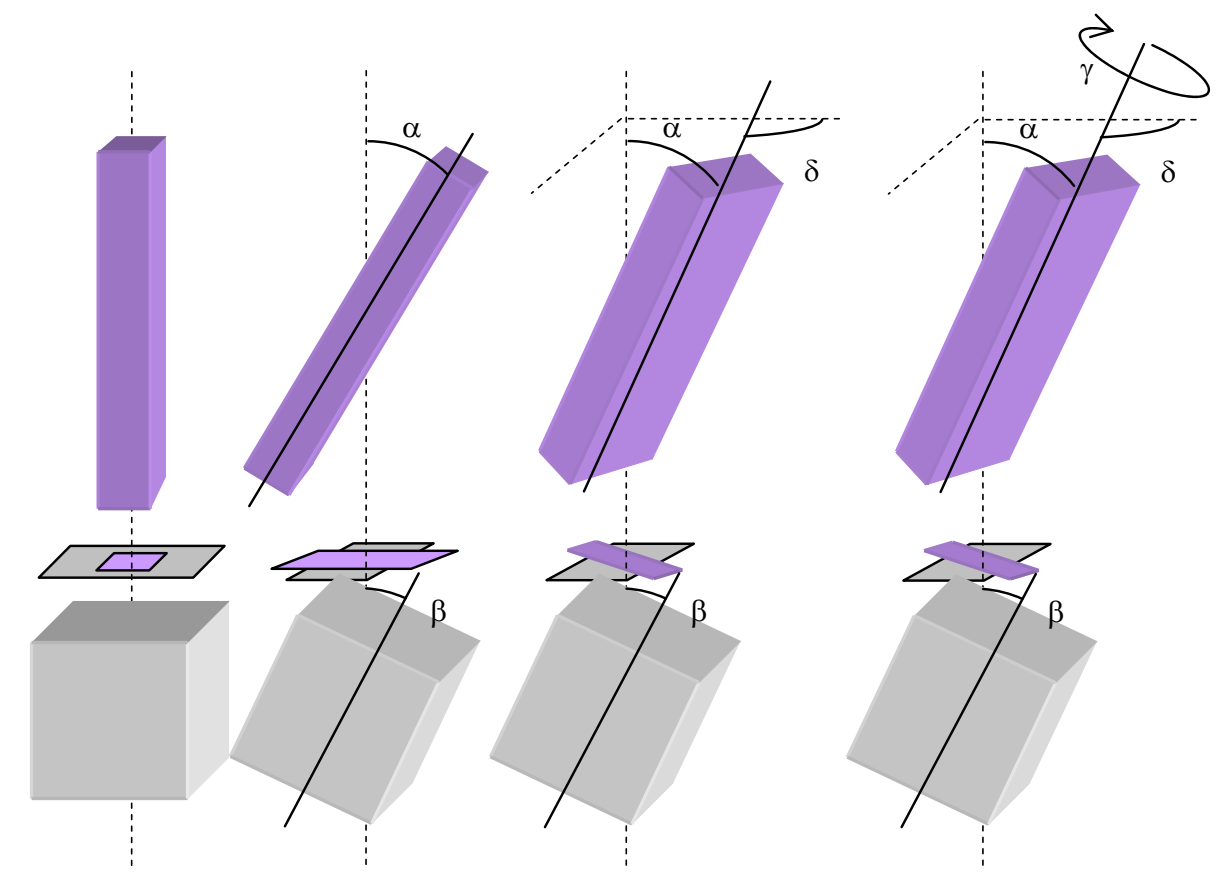

Figure S2: Angles which describe the relative orientation of guest and host relative to the collision axis.

Displaced collisions can always be transformed into collisions along a common axis by rotation of guest and host:

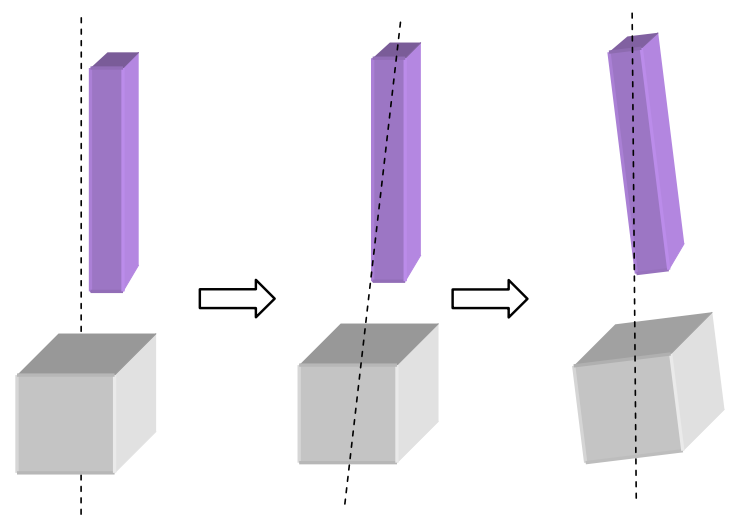

Figure S3: Displaced (non central) collisions. 


\section{Conditions for successful collisions}

A collision is taken as successful when the projected outline of the guest is smaller than the opening of the cavity as seen from the guest:

For this the vertices of the guest and host are projected along the common axis into a hypothetical intermediate plane (see figs S2 and S4). Then the projected outline of the guest is compared to the projection of the cavity's openings, taking into account the relative inclinations and rotations.
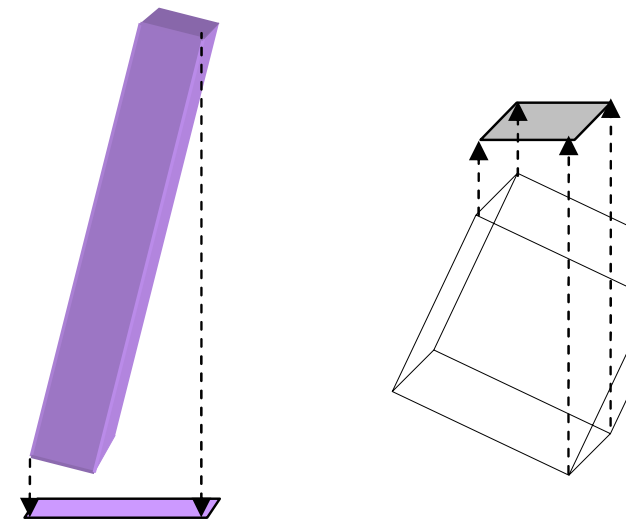

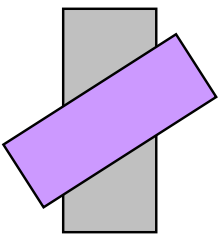

not successful

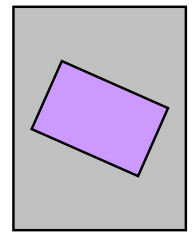

successful

Figure S4: Projection of guest and host to a hypothetical intermediate plane..

\section{Projections}

The widths of the projections were calculated differently for host and guest:

Host: The projection of the host's square prism depends only on the angle $\beta$ :

$$
a_{C D}=\operatorname{Max}(b \cos \beta-L \sin \beta, 0)
$$

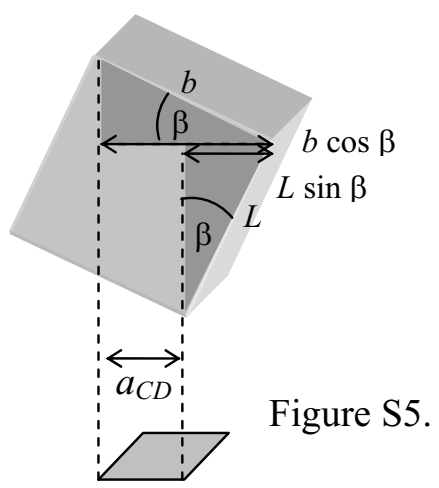

Guest: The projection of the rectangular prism of the guest depends on three angles. The projection was determined by rotation of a cuboid of given dimensions by the angles $\alpha, \delta$ and $\gamma$ (affine rotation).

\section{Procedure}

The angles $\alpha, \beta, \delta$ and $\gamma$ were varied systematically with steps $\Delta \alpha, \Delta \beta, \Delta \delta$ and $\Delta \gamma$ in the intervals given below. The number of orientations which correspond to successful collisions $N_{R}$ and the total number of orientations $N$ were counted. This procedure was programmed with the software package Mathematica (Wolfram Research, Champaign USA). 


\section{Intervals}

Due to the symmetry of the system the angles have only to be varied between 0 and $90^{\circ}$, that is only one eighth of the full sphere has to be analyzed. The ratio $\mathrm{N}_{\mathrm{R}} / \mathrm{N}$ is the same in all other segments.

Some care has to be taken with the limits of the interval. The case $\alpha=\beta=0$ was counted as a single successful collision.

$$
0<\alpha<\pi / 2 ; 0<\beta<\pi / 2 ; 0 \leq \delta<\pi / 2 ; 0 \leq \gamma<\pi / 2
$$

\section{Typical molecular dimensions}

The following width were used in the simulations. The values were estimated from three dimensional models and coincide well with literature values. ( $\beta$-CD: Szejtli, J. Chem. Rev., 1998, 98(5), 1743)

\begin{tabular}{|l|l|l|l|}
\hline & $\mathbf{b} / \AA$ & $\mathbf{c} / \boldsymbol{A}$ & $\mathbf{L} / \AA$ \\
\hline $\mathrm{PY}$ & $\approx 6$ & $\approx 4$ & $\approx 14$ \\
\hline $\mathrm{PB}$ & $\approx 6$ & $\approx 5$ & $\approx 14$ \\
\hline$\beta-\mathrm{CD}$ & $6-7$ & $6-7$ & $\approx 8$ \\
\hline
\end{tabular}

\section{Results}

a) Variation of the "gap" between guest and host:

the width of the cavity of the host $b_{C D}$ is held fixed at $6.5 \AA$, and the longer width $b_{P}$ of the guest is varied:

\begin{tabular}{|l|l|l|l|l}
$\boldsymbol{b}_{P,} \boldsymbol{c}_{P}, \boldsymbol{L}_{\boldsymbol{P}} / \boldsymbol{\AA}$ & $\boldsymbol{b}_{C D}, \boldsymbol{L}_{C D} / \AA$ & $\Delta \alpha=\Delta \beta, \Delta \gamma, \Delta \delta$ & $\boldsymbol{N}, \boldsymbol{N}_{\boldsymbol{R}}$, & $\boldsymbol{f}_{\boldsymbol{R}}=\boldsymbol{N}_{\boldsymbol{R}} / \boldsymbol{N}$ \\
\hline $\mathbf{6}, 4,14$ & $6.5,8$ & $\pi / 100, \pi / 100, \pi / 60$ & 3601501,1036 & $\mathbf{0 . 0 0 0 3}$ \\
\hline $\mathbf{5}, 4,14$ & $6.5,8$ & & 3601501,6775 & $\mathbf{0 . 0 0 2}$
\end{tabular}

Reducing the width $b_{P}$ from $6 \AA$ to $5 \AA$ increments $\mathrm{f}_{\mathrm{R}}$ by a factor of nearly ten.

b) Variation of the smaller width $c_{P}$ of the guest:

\begin{tabular}{|l|l|l|l|l|}
\hline $\boldsymbol{b}_{P,} \boldsymbol{c}_{P}, \boldsymbol{L}_{\boldsymbol{P}} / \boldsymbol{\AA}$ & $\boldsymbol{b}_{C D}, \boldsymbol{L}_{C D} / \AA$ & $\Delta \alpha=\Delta \boldsymbol{\beta}, \Delta \gamma, \Delta \boldsymbol{\delta}$ & $\boldsymbol{N}, \boldsymbol{N}_{\boldsymbol{R}}$, & $\boldsymbol{f}_{\boldsymbol{R}}=\boldsymbol{N}_{\boldsymbol{R}} / \boldsymbol{N}$ \\
\hline $6, \mathbf{5}, 14$ & $6.5,8$ & $\pi / 100, \pi / 100, \pi / 60$ & 3601501,325 & $\mathbf{0 . 0 0 0 1}$ \\
\hline $6, \mathbf{4}, 14$ & $6.5,8$ & & 3601501,1036 & $\mathbf{0 . 0 0 0 3}$ \\
\hline $6, \mathbf{3}, 14$ & $6.5,8$ & & 3601501,2418 & $\mathbf{0 . 0 0 0 7}$ \\
\hline
\end{tabular}

Reducing the width $c_{P}$ by $1 \AA$ increments $\mathrm{f}_{\mathrm{R}}$ only by a factor $2-3$. 